\title{
A randomized controlled study comparing microsurgical clipping via the keyhole approach, conventional craniotomy, and endovascular coiling for ruptured anterior circulation aneurysms
}

Qing Zhu ( $\square$ suzhouneurosurgeon@126.com )

Second Affiliated Hospital of Soochow University https://orcid.org/0000-0003-4345-9754

Qing Lan

Second Affiliated Hospital of Soochow University https://orcid.org/0000-0002-8468-4942

Ailin Chen

Second Affiliated Hospital of Soochow University

\section{Research Article}

Keywords: intracranial aneurysm, surgical approach, keyhole, therapy, endovascular, therapy, clip

Posted Date: November 18th, 2021

DOI: https://doi.org/10.21203/rs.3.rs-1072688/v1

License: @ (i) This work is licensed under a Creative Commons Attribution 4.0 International License.

Read Full License 


\section{Abstract}

Objective: Few prospective randomized controlled studies have investigated the efficacy of endovascular treatment and microsurgical clipping of intracranial aneurysms, especially via microsurgical keyhole approach. We compared the efficacy of three techniques in treating patients with ruptured anterior circulation aneurysms to provide surgeons with a more objective basis for treatment selection.

Methods: 150 patients with ruptured anterior circulation aneurysms were randomly assigned to endovascular treatment, conventional craniotomy, and keyhole approach groups. Aneurysm occlusion, surgical time, hospitalization time, hospitalization expenses, and surgical complications were compared between groups.

Results: The complete occlusion rates of aneurysms at discharge were $90 \%$ in the endovascular group, $94 \%$ in the conventional group, and $96 \%$ in the keyhole group. No significant differences in complete occlusion rates or Glasgow Outcome Scale scores were found between groups. In the keyhole approach, conventional craniotomy, and endovascular groups, the overall surgical times were $161.78 \pm 34.51 \mathrm{~min}$, $201.55 \pm 38.79 \mathrm{~min}$, and $85.86 \pm 58.57 \mathrm{~min}$, respectively; the hospitalization times were $11.42 \pm 6.64 \mathrm{~d}$, $18.03 \pm 7.14 \mathrm{~d}$, and $10.57 \pm 8.67 \mathrm{~d}$; hospitalization expenses were 10574.25 $\pm 4154.25 \mathrm{USD}$, 13214.54 \pm 5487.65 USD, and 20134.58 \pm 6587.61 USD; and the incidence rates of postoperative complications such as intracranial infection, cerebral vasospasm, hydrocephalus, intracranial hematoma, and epilepsy were $8 \%, 28 \%$, and $20 \%$.

Conclusions: Endovascular coiling and the microsurgical keyhole approach have the advantages of simple execution, time savings, and short hospitalization. Microsurgical clipping of intracranial aneurysms needs to be updated to a minimally invasive procedure to maintain its complementary value with endovascular treatment.

Clinical trial registration: The study has been retrospectively registered in clinicaltrial.org (NCT05049564) in Sep. 8th, 2021.

\section{Introduction}

Since Perneczky published a monograph on the keyhole approach [30], the minimally invasive keyhole approach has been applied to the treatment of not only unruptured anterior circulation aneurysms but also ruptured aneurysms, large and giant aneurysms, and posterior circulation aneurysms $[2,8,19,27]$. Some reports even discuss bilateral intracranial aneurysm surgery using a unilateral keyhole approach [38]. Although both endovascular treatment and microsurgical clipping with the keyhole approach have shown good therapeutic effects, few prospective randomized controlled trials have compared the efficacy of these two minimally invasive techniques in the treatment of intracranial aneurysm $[24,26]$.

In this study, patients with ruptured anterior circulation aneurysms, which are easy to standardize, were the study subjects, and a randomized controlled analysis comparing the keyhole approach, conventional 
craniotomy, and endovascular treatment was performed to clarify the advantages, disadvantages, and outcomes of these three techniques.

\section{Materials And Methods}

Inclusion criteria: 1. Patients diagnosed with a single intracranial anterior circulation aneurysm by computed tomography angiography (CTA) or digital subtraction angiography (DSA); 2 . patients with subarachnoid hemorrhage diagnosed by computed tomography (CT) due to aneurysm rupture, which was confirmed during surgery; 3 . patients without indications for decompressive craniectomy (Hunt-Hess grade $\leq 4$, Glasgow Coma Scale (GCS) score $\geq 7$, no pupil dilation, and CT showing a midline shift $<5$ $\mathrm{mm}$ ); and 4. patients with aneurysms suitable for both endovascular treatment and microsurgical clipping. From January 2013 to September 2017, a total of 150 consecutive patients meeting the inclusion criteria were included in this study.

Exclusion criteria: 1 . Lack of consent to inclusion by the patient or the patient's family; 2 . unruptured anterior circulation aneurysms; 3 . multiple intracranial aneurysms; and 4. serious concomitant diseases contraindicating treatment.

Groups: Endovascular treatment group: Patients underwent endovascular treatment with embolization or stent-assisted embolization. Keyhole approach group: Patients underwent microsurgical clipping via the keyhole approach. Conventional craniotomy group: Patients underwent microsurgical clipping through traditional bone flap craniotomy. No significant differences in clinical data were found between the groups.

Ethics: This study was approved by the Ethics Committee of the Second Affiliated Hospital of Soochow University. The patients were randomly grouped according to admission time once they or their families provided written consent. The study has been registered in clinicaltrial.org (NCT05049564).

Surgeons: Our surgeons learn both the endovascular and microsurgical keyhole treatment methods. After 3 years of extensive experience in applying these techniques, the surgeons could perform the surgeries in this randomized controlled study.

Patients: From January 2013 to September 2017, 150 consecutive patients with ruptured anterior circulation aneurysms meeting the inclusion criteria were included in this study (Table 1).

\section{Treatment methods}

\section{Microsurgical approaches}

The keyhole approach involved a 4-cm skin incision and a cranial hole with a diameter of 2-2.5 cm, and the internal skull plate at the front edge of the operative field was ground to expand the surgical field of view. After surgery, the skull flap was repaired, and no drainage tube was placed in the surgical region. This study used the supraorbital keyhole approach, pterional keyhole approach, and trans-longitudinal 
fissure keyhole approach [17]. Conventional craniotomy followed the Yasargil pterional approach and interhemispheric approach, and the surgical incisions were approximately $15 \mathrm{~cm}$ [6] (Figure 1).

\section{Microsurgical procedure}

First, the Sylvian cistern, carotid cistern, prechiasmatic cistern, and other brain cisterns were opened to fully release the bloody cerebrospinal fluid and separate and expose the parent arteries. For patients with high intracranial pressure or severe subarachnoid hemorrhage, lamina terminalis fenestration was performed or the Liliequist membrane was opened to further release cerebrospinal fluid. After separating the aneurysm neck, different types of aneurysm clips were selected to clip the aneurysm neck according to the direction, size, and approach angle of the aneurysm. When an aneurysm ruptured during surgery, two aspirators were used to control the bleeding. In the keyhole group, a lumbar cistern drainage tube was placed after surgery, while in the conventional group, subdural and epidural drainage tubes were placed after surgery, and bloody cerebrospinal fluid was drained continuously for 3-5 days.

\section{Endovascular treatment}

The Seldinger method was used to establish the femoral artery approach, and the whole body was heparinized. A 6-F guiding catheter was placed at the level of the $\mathrm{C} 2$ vertebral body of the internal carotid artery on the side of the lesion. According to the location, orientation, and volume of the aneurysm, the tip of the microcatheter was shaped by steam and inserted into the aneurysm cavity under the guidance of a microguide wire, and detachable coils were packed one by one until the aneurysm cavity was blocked off. If the coil could not be stabilized in the aneurysm cavity, a stent was used to protect the parent artery. Plavix (75 mg orally qd) was prescribed for up to 6 weeks after the surgery, and aspirin ( $100 \mathrm{mg}$ orally qd) was prescribed for life.

\section{Follow-up after surgery}

For patients in the microsurgery group, CTA or DSA was performed at the first reexamination within 1 week after surgery and again 6 months after surgery. Patients in the endovascular group were reexamined at 6 months after surgery. The Glasgow Outcome Scale (GOS) was used to evaluate the prognosis during discharge and follow-up.

\section{Statistical analysis}

A Student's t-test was used to compare the age, time and cost. A $X^{2}$ test was used for sex, grade, operative period, aneurysm diameter, aneurysmal rupture, incidence of complications, and GOS score. All data were analyzed by SPSS 21.0 software (IBM, Chicago, USA). P $<0.05$ was set as statistically significant.

\section{Results}

\section{Aneurysm treatment}


Among the 150 patients (Table 2), 100 received microsurgical treatment, including 50 by the keyhole approach (24 patients via the supraorbital keyhole approach, 22 patients via the pterional keyhole approach, four patients via the interhemispheric keyhole approach) and 50 by conventional craniotomy (46 patients via the Yasargil pterional approach, four patients via the interhemispheric approach). During the clipping of these 100 aneurysms, 21 aneurysms ruptured, including 10 in the keyhole group and 11 in the conventional group, and were properly clipped after two aspirators were used to control the bleeding; no blood was transfused during the surgery. No significant difference in treatment time (the subdural manipulation time) was noted between the keyhole group and the conventional group ( $P=0.81)$. The craniotomy and closure times (the time from scalp incision to completion of the dural incision + the time from suture of the dura to completion of the scalp suture) were $60.54 \pm 17.54$ minutes in the keyhole group and $98.54 \pm 24.87$ minutes in the conventional group $(P<0.001)$.

Among the 50 aneurysms in the endovascular group, 35 aneurysms were simply embolized, and 15 aneurysms were assisted by stents. Eight aneurysms ruptured during surgery, and hemostasis was achieved after fast coil packing. The surgical time in the endovascular treatment group was significantly shorter than that in the two microsurgery groups $(P<0.001)$.

\section{Aneurysm occlusion}

Among the 100 patients treated by microsurgery, one patient died due to severe lung infection, and the other 99 patients were reexamined with head CTA or DSA after surgery and followed up 6 months later. Two patients with anterior communicating artery aneurysms in the keyhole group (4\%) and two patients with posterior communicating artery aneurysms and one patient with an anterior communicating artery aneurysm in the conventional group (6\%) had a small residual aneurysm neck after surgery. For one of the patients in the keyhole approach group with an anterior communicating artery aneurysm, the residual aneurysm neck had completely disappeared at the 6-month follow-up exam. Among the 50 patients with endovascular treatment, Raymond grade 1 occlusion was achieved in 45 aneurysms, and Raymond grade 2 occlusion was achieved in five aneurysms after surgery (10\%); three of these patients died, and 47 patients were reexamined with DSA 6 months after surgery. Three of the 32 patients with simple coil embolization were found to have aneurysm neck recurrence.

\section{Length of postoperative hospitalization and medical expenses}

The length of postoperative hospitalization in the keyhole group was $11.42 \pm 6.64$ days, which was significantly shorter than that in the conventional group $(18.03 \pm 7.14$ days, $\mathrm{P}<0.001)$ but was comparable to that in the endovascular group $(10.57 \pm 8.67$ days, $\mathrm{P}=0.32)$. The total hospitalization cost in the keyhole group was (10574.25 \pm 4154.25 USD), which was lower than that in the conventional group (13214.54 \pm 5487.65 USD, $P=0.03$ ), while the total hospitalization cost in the endovascular group was 20134.58 \pm 6587.61 USD, which was significantly higher than that in the other two microsurgery groups $(\mathrm{P}<0.001)$.

\section{Complications}


As shown in Table 2, in the keyhole, conventional, and endovascular groups, the incidence rates of postoperative complications, including intracranial infection, cerebral vasospasm, hydrocephalus, intracranial hematoma, and epilepsy, were $8 \%, 28 \%$, and $20 \%$, respectively; the reoperation rates were $2 \%$ (1/50, decompressive craniectomy due to cerebral infarction caused by cerebral vasospasm), $12 \%$ (6/50, one patient underwent removal of delayed intracerebral hematomas, two patients underwent decompressive craniectomy, three patients underwent ventricular-peritoneal shunting), and $26 \%(13 / 50$, four patients underwent ventricular drainage, which was changed to a ventricular-peritoneal shunt in one patient after extubation, four patients underwent traditional bone flap craniectomy due to increased intracranial hemorrhage, two patients underwent decompressive craniectomy, three patients underwent ventricular-peritoneal shunting); and the mortality rates were $2 \%$ ( $1 / 50$, lung infection), $0 \%$, and $6 \%(3 / 50$, intraoperative aneurysm rupture).

\section{Discussion}

\section{Feasibility of aneurysm clipping via the keyhole approach}

This study showed that the operation time in epidural in the keyhole group was significantly shorter than that in the conventional craniotomy group, but no significant difference in the aneurysm clipping (intracranial manipulation) time was observed; this finding indicated that the keyhole approach does not prolong surgery due to the narrow bone hole and that aneurysm clipping with the traditional surgical approach can be carried out smoothly using the keyhole approach. The reason is that with the keyhole approach, only extra space that is basically unnecessary for surgery is removed, while the key operative sites are retained [32]. Intracranial aneurysms are located in the subarachnoid space, and the operating channels for the keyhole approach enter through the gap between the brain tissue and the dura. A gap measuring $2.5 \mathrm{~cm}$ can fully meet the exposure requirements of deep structure surgery, and excessive brain tissue traction may cause unnecessary contusion of brain tissue. Designing the surgical approach before surgery is important to facilitate aneurysm clipping. If the aneurysm is oriented laterally, the anterior supraorbital keyhole approach can expose the aneurysm neck well; however, for dorsally oriented aneurysms, fenestration or right-angle clips may be required during the anterior approach (the parent artery may partially obscure the aneurysm clips), and under the lateral pterional keyhole approach, the aneurysm neck can be clearly exposed [18]. In addition to this study, we have successfully performed the keyhole approach on complex aneurysms (large or giant aneurysms, aneurysms in the clinoid segment, and posterior circulation aneurysms), which fully demonstrates that the minimally invasive keyhole approach is effective and feasible for aneurysm clipping [15,16,21]. If necessary, simulated operations can also be performed preoperatively by printing a 3D model of intracranial aneurysms to plan or verify the feasibility of the keyhole approach $[14,20]$.

\section{Effectiveness of aneurysm clipping by the keyhole approach}

In this study, aneurysm clipping was successful in all 50 patients who underwent keyhole approach surgery, 44 of whom underwent surgery in the acute stage, while $6 / 50$ patients underwent surgery under 
the so-called vasospasm period, 10/50 patients experienced intraoperative aneurysm rupture, and $5 / 50$ patients had a Hunt-Hess grade of IV. Therefore, for patients without cerebral hernia before surgery, intracranial pressure can be effectively reduced, and sufficient intracranial operative space is provided through the release of cerebrospinal fluid and the use of dehydrating agents during surgery. Even if the aneurysm ruptures intraoperatively, the bleeding can be quickly controlled with two aspirators, and then, aneurysm clipping can be completed $[4,28,37]$. One patient with Hunt-Hess grade IV underwent decompressive craniectomy 2 days after surgery in the acute stage because of high intracranial pressure caused by cerebral vasospasm and recovered well after hyperbaric oxygen therapy, indicating that in some patients with severe subarachnoid hemorrhage and poor preoperative grading, traditional bone flap craniotomy and decompressive craniectomy should be performed early depending on their conditions.

\section{Comparison of the keyhole approach and conventional craniotomy}

The duration of epidural operation in the keyhole group was on average 40 minutes shorter than that in the conventional craniotomy group, and the hospitalization time was 1 week shorter, enabling the surgeon to spend more time and energy on the treatment of intracranial lesions and allowing the patient to recover and return home as soon as possible. Therefore, the treatment costs were also lower.

The greatest difference between the keyhole approach and conventional craniotomy is the extent of the surgical trauma and related injuries. Some people may think that a larger surgical incision is not harmful to the patient, but in fact, the psychological fortitude of the patient and the postoperative impact are substantially affected by the incision size. Two patients in the conventional craniotomy group had postoperative intracerebral hematoma due to brain traction and contusion. In contrast, the small bone holes of the keyhole approach can limit the traction on brain tissue. In addition, the incidence of postoperative infections and epilepsy in the conventional group was higher than that in the keyhole group. One patient had undergone clipping of a posterior communicating artery aneurysm through conventional craniotomy in another hospital one year previously. After surgery, the patient had obvious feelings of discomfort and then suffered from temporal muscle atrophy with a sunken appearance after discharge. The patient was admitted to our department because of rupture of a contralateral posterior communicating artery aneurysm and was treated by the pterional keyhole approach (Figure $1 \mathrm{D}-\mathrm{E}$ ). After surgery, the patient felt well and happy, with no defects in appearance, and considered that the keyhole approach was quite different from conventional craniotomy. Many patients with aneurysms choose interventional surgery because they are afraid of conventional craniotomy. The minimally invasive nature of the keyhole approach can increase their acceptance of microsurgery $[1,7,31]$.

Indeed, small bone holes are required with minimally invasive keyhole approach, which may cause some discomfort to surgeons who are familiar with conventional craniotomy. In fact, in the past, when conventional craniotomy was performed, the actual operating space was fairly similar to that of the keyhole approach, and experienced aneurysm surgeons do not experience difficulty when trying different surgical approaches. Young surgeons can quickly master the keyhole approach after training in microanatomy or under the guidance of surgeons familiar with the keyhole approach. The disadvantage 
of the keyhole approach is that it is limited to a narrow cranial hole, and changing the direction of the aneurysm clip during the surgery is difficult $[25,29,34]$. Under a fixed operating angle, surgeons can only rely on different aneurysm clips and adjustment of the operating table to reach the best clipping angle. For some complex aneurysms, if multiangle observation or clipping is required, conventional craniotomy is favorable.

\section{Comparison of the keyhole approach and endovascular treatment}

For interventional therapy, no open injury or blood transfusion is required, and the incidence of postoperative infection and epilepsy is low $[9,10,12]$. This advantage of endovascular treatment was also confirmed by this study.

The response to aneurysm rupture during surgery is one of the key factors affecting the efficacy of aneurysm surgery. The countermeasures in conventional craniotomy are all suitable for the keyhole approach $[11,13]$. In this study, 10 aneurysms in the keyhole group and 11 aneurysms in the conventional craniotomy group ruptured during surgery, and the bleeding was always quickly and properly controlled, without complications caused by poor management of ruptured aneurysms. However, aneurysm rupture during endovascular treatment is very different and is often fatal, and no rapid and effective treatment methods are available $[33,36]$. In the endovascular treatment group of this study, although eight patients with intraoperative aneurysm rupture received rapid hemostasis by rapid coil packing, four had increased intracranial pressure and brain swelling after surgery and underwent emergency conventional craniotomy. Among them, two patients died, resulting in a mortality rate of intraoperative rupture of $25 \%(2 / 8)$.

The rapid development of endovascular treatment techniques has expanded the indications for the treatment of intracranial aneurysms [25], but at present, some aneurysms are better suited for surgical clipping. For example, for wide-necked aneurysms, no subsequent treatment of double antiplatelet due to stent implantation during endovascular treatment is required after surgical clipping; for patients with obvious intracranial hemorrhage, the hematoma can be removed and decompressed at the time of surgical clipping, or the hemorrhagic cerebrospinal fluid can be fully released to reduce the incidence of vasospasm and hydrocephalus; clipping and shaping of giant aneurysms can achieve a great effect; and surgical clipping is still a better option for patients with intracranial arterial tortuosity and for aneurysms that are hard to reach with the catheter guide wire $[5,22,23,35]$. For long-term efficacy, endovascular treatment still has a certain recurrence rate. In this study, DSA at the 6-month follow-up showed that three of the 35 aneurysms with simple coils had recurrence (8.57\%), even if they reached Raymond grade I occlusion after the surgery. Microsurgical clipping of intracranial aneurysms is still a key treatment technique, but we also need to adapt to new advancements and make aneurysm clipping more minimally invasive, for which the keyhole approach is currently the most representative, optimal choice $[3,17]$.

\section{Limitations}

This study is a single-center study with an insufficient sample size. The findings require further confirmation by multicenter studies with large samples. 


\section{Conclusions}

This study showed that both surgical clipping and interventional therapy have good therapeutic effects, but minimally invasive surgery should be the first choice and future direction for intracranial aneurysm treatment. Surgical training promoting mastery of both surgical clipping and interventional therapy is beneficial for perfecting intracranial aneurysm treatment and will enable formulation of the best individualized treatment plan for each patient [13]. In the future, large-scale multicenter case studies should be designed to verify the results of this study.

\section{Declarations}

Funding: This study was funded by National Key Research and Development Program of China (2016YFC1300701) and Foundation of Suzhou Key Laboratory of Minimally Invasive Neurosurgery (SZS2021262).

Conflict of interest: The authors declare that they have no conflicts of interest.

Availability of data and material: Not applicable

Code availability: Not applicable

Ethics approval: Approval was obtained from the ethics committee of Second Affiliated Hospital of Soochow University. The procedures used in this study adhere to the tenets of the Declaration of Helsinki.

Consent to participate: Informed consent was obtained from all individual participants included in the study.

Consent for publication: The authors affirm that human research participants provided informed consent for publication of the images in Figure $1 \mathrm{a} \& 1 \mathrm{~b}, 1 \mathrm{c}, 1 \mathrm{~d} \& 1 \mathrm{e}$.

Authors' contributions: All authors contributed to the study conception and design. Material preparation, data collection and analysis were performed by Qing Zhu, Qing Lan and Ailin Chen. The first draft of the manuscript was written by Qing Zhu and all authors commented on previous versions of the manuscript. All authors read and approved the final manuscript.

\section{References}

1. Beseoglu K, Lodes S, Stummer W, Steiger HJ, Hänggi D (2011) The transorbital keyhole approach: early and long-term outcome analysis of approach-related morbidity and cosmetic results. Technical note. J Neurosurg 114(3):852-856

2. Chalouhi N, Jabbour P, Ibrahim I, Starke RM, Younes P, Hage GE, Samaha E (2013) Surgical treatment of ruptured anterior circulation aneurysms: comparison of pterional and supraorbital keyhole approaches. Neurosurgery 72(3):437-441 
3. Chen LG, Chen SD, Huang GF et al (2017) Application of keyhole microneurosurgery in China. Chin Med J (Engl) 130(16):1987-1994

4. Chiappini A, Marchi F, Reinert M, Robert T (2018) Supraorbital approach through eyebrow skin incision for aneurysm clipping: how I do it. Acta Neurochir (Wien) 160(6):1155-1158

5. Choi JH, Lee KS, Kim BS, Shin YS (2020) Treatment outcomes of large and giant intracranial aneurysms according to various treatment modalities. Acta Neurochir (Wien) 162(11):2745-2752

6. Davies JM, Lawton MT (2014) Advances in open microsurgery for cerebral aneurysms. Neurosurgery 74 Suppl1:S7-16. https://doi.org/10.1227/NEU.0000000000000193

7. Dlouhy BJ, Chae MP, Teo C (2015) The supraorbital eyebrow approach in children: clinical outcomes, cosmetic results, and complications. J Neurosurg Pediatr 15(1):12-19

8. Fische G, Stadie A, Reisch R, Hopf NJ, Fries G, Böcher-Schwarz H, van Lindert E, Ungersböck K, Knosp E, Oertel J, Perneczky A (2011) The keyhole concept in aneurysm surgery: results of the past 20 years. Neurosurgery 68(1 Suppl Operative):45-51

9. Fotakopoulos G, Andrade-Barazarte H, Tjahjadi M, Goehre F, Hernesniemi J (2021) Clipping versus coiling in ruptured basilar apex aneurysms: a meta-analysis. Turk Neurosurg 31(3):301-309

10. Fotakopoulos G, Tsianaka E, Fountas K, Makris D, Spyrou M, Hernesniemi J (2017) Clipping versus coiling in anterior circulation ruptured intracranial aneurysms: a meta-analysis. World Neurosurg 104:482-488

11. Houkin K, Kuroda S, Takahashi A, Takikawa S, Ishikawa T, Yoshimoto T, Itamato K (1999) Intraoperative premature rupture of the cerebral aneurysms. Analysis of the causes and management. Acta Neurochir (Wien) 141(12):1255-1263

12. Jiang $Z$, Chen $Y$, Zeng C, Feng J, Wan $Y$, Zhang $X$ (2020) Neurosurgical clipping versus endovascular coiling for patients with intracranial aneurysms: a systematic review and meta-analysis. World Neurosurg 138:e191-e222

13. Lan Q (2006) Clinical application of keyhole techniques in minimally invasive neurosurgery. Chin Med J (Engl) 119(16):1327-1330

14. Lan Q, Chen A, Zhang T, Li G, Zhu Q, Fan X, Ma C, Xu T (2016) Development of three-dimensional printed craniocerebral models for simulated neurosurgery. World Neurosurg 91:434-442

15. Lan Q, Chen J, Qian ZY, Zhang QB, Huang Q (2007) Microsurgical treatment of complex intracranial aneurysms via keyhole approaches. Zhonghua Yi Xue Za Zhi 87(13):872-876

16. Lan Q, Gong Z, Kang D, Zhang H, Qian Z, Chen J, Huang Q (2006) Microsurgical experience with keyhole operations on intracranial aneurysms.Surg Neurol66 Suppl 1:S2-9. https://doi.org/10.1016/j.surneu.2006.06.039

17. Lan Q, Sughrue M, Hopf NJ et al (2021) International expert consensus statement about methods and indications for keyhole microneurosurgery from International Society on Minimally Invasive Neurosurgery. Neurosurg Rev 44(1):1-17

18. Lan Q, Zhang H, Zhu Q, Chen A, Chen Y, Xu L, Wang Z, Yuan L, Liu S (2017) Keyhole approach for clipping intracranial aneurysm: comparison of supraorbital and pterional keyhole approach. World 
Neurosurg 102:350-359

19. Lan Q, Zhu Q, Li G (2015) Microsurgical treatment of posterior cerebral circulation aneurysms via keyhole approaches. World Neurosurg 84(6):1758-1764

20. Lan Q, Zhu Q, Xu L, Xu T (2020) Application of 3D-printed craniocerebral model in simulated surgery for complex intracranial lesions. World Neurosurg 134:e761-e770

21. Ma Y, Lan Q (2011) Supraorbital keyhole approach to upper basilar artery aneurysms via the opticocarotid window: a cadaveric anatomic study and preliminary application. Minim Invasive Neurosurg 54(5-6):228-235

22. Mascitelli JR, Lawton MT, Hendricks BK, Nakaji P, Zabramski JM, Spetzler RF (2019) Analysis of wideneck aneurysms in the Barrow Ruptured Aneurysm Trial. Neurosurgery 85(5):622-631

23. Mascitelli JR, Oermann EK, De Leacy RA, Moyle H, Mocco J, Patel AB (2015) Predictors of treatment failure following coil embolization of intracranial aneurysms. J Clin Neurosci 22(8):1275-1128

24. Molyneux AJ, Kerr RS, Yu LM, Clarke M, Sneade M, Yarnold JA, Sandercock P, International Subarachnoid Aneurysm Trial (ISAT) Collaborative Group (2005) International Subarachnoid Aneurysm Trial (ISAT) of neurosurgical clipping versus endovascular coiling in 2143 patients with ruptured intracranial aneurysms: a randomized comparison of effects on survival, dependency, seizures, rebleeding, subgroups, and aneurysm occlusion. Lancet 366(9488):809-817

25. Mori K (2014) Keyhole concept in cerebral aneurysm clipping and tumor removal by the supraciliary lateral supraorbital approach. Asian J Neurosurg 9(1):14-20

26. Paladino J, Mrak G, Miklić P, Jednacak H, Mihaljević D (2005) The keyhole concept in aneurysm surgery - a comparative study: keyhole versus standard craniotomy. Minim Invasive Neurosurg 48(5):251-258

27. Park J (2014) Superciliary keyhole approach for unruptured anterior circulation aneurysms: surgical technique, indications, and contraindications. J Korean Neurosurg 56(5):371-374

28. Park J (2020) Supraorbital keyhole approach for intracranial aneurysms: transitioning from concerns to confidence. J Korean Neruosurg Soc 63(1):4-13

29. Park JS, Kim H, Baik MW, Park IS (2018) Risk factor analysis for poor outcomes in supraorbital keyhole aneurysm clipping for ruptured anterior circulation aneurysms. World Neurosurg 111:e386e394

30. Perneczky A, Muller-Forell W, van Linert E, Fries G (1999) Keyhole concept in neurosurgery with endoscopic-assisted microsurgery and case studies. Thieme

31. Reisch R, Marcus HJ, Hugelshofer M, Koechlin NO, Stadie A, Kockro RA (2014) Patients' cosmetic satisfaction, pain, and functional outcomes after supraorbital craniotomy through an eyebrow incision. J Neurosurg 121(3):730-734

32. Reisch R, Stadie A, Kockro RA, Hopf N (2013) The keyhole concept in neurosurgery.World Neurosurg79(2 Suppl):S17.e9-13. https://doi.org/10.1016/j.wneu.2012.02.024

33. Sharma DP, Singh D, Jagetia A, Singh H, Tandon M, Ganjoo P (2011) Intra procedure rupture of intracranial aneurysm during endovascular coiling: neurosurgeons' experience and review of the 
literature. Neurol India 59(5):690-695

34. van Lindert E, Perneczky A, Fries G, Pierangeli E (1998) The supraorbital keyhole approach to supratentorial aneurysm: concept and technique. Surg Neurol 49(5):481-489

35. Xia ZW, Liu XM, Wang JY, Cao H, Chen FH, Huang J, Li QZ, Fan SS, Jiang B, Chen ZG, Cheng Q (2017) Coiling is not superior to clipping in patients with high-grade aneurismal subarachnoid hemorrhage: systematic review and meta-analysis. World Neurosurg 98:411-420

36. Yamagami K, Hatano T, Nakahara I, Ishii A, Ando M, Chihara H, Ogura T, Suzuki K, Kondo D, Kamata T, Higashi E, Sakai S, Sakamoto H, lihara K, Nagata I (2020) Long-term outcomes after intraprocedural aneurysm rupture during coil embolization of unruptured intracranial aneurysms. World Neurosurg 134:e289-e297

37. Yamahata H, Tokimura H, Tajitsu K, Tsuchiya M, Taniguchi A, Hirabaru M, Takayama K, Shinsato T, Hirayama T, Arita K (2014) Efficacy and safety of the pterional keyhole approach for the treatment of anterior circulation aneurysms. Neurosurg Rev 37(4):629-636

38. Yu LH, Shang-guan HC, Chen GR, Zheng SF, Lin YX, Lin ZY, Yao PS, Kang DZ (2017) Monolateral pterional keyhole approaches to bilateral cerebral aneurysms: anatomy and clinical application. World Neurosurg 108:572-580

\section{Tables}


Table 1. Total Number of Enrolled Patients and Operative Times

\section{Keyhole approach group}

50 patients

$50.24 \pm 13.14$

Mean age (years) ${ }^{\mathrm{a}}$

Sex $(\text { male/female })^{\mathrm{b}} \quad$ 18/32

Hunt-Hess grade

$\begin{array}{ll}\Pi^{\mathrm{c}} & 35 \\ \mathrm{II}^{\mathrm{d}} & 10 \\ \mathrm{IV}^{\mathrm{e}} & 5\end{array}$

Fisher grade

$$
\text { II }^{f}
$$

III

IVh

Operative time

Acute stage $(<3 \mathrm{~d})^{\mathrm{i}} \quad 44$

Subacute stage (3- 6

$14 d)^{j}$

Chronic stage $(>14 \quad 0$

d)

28

30

29

10

8

12

10

33

13

14

45

12

45

44

5

6

Maximum diameter of aneurysm $₫ \mathrm{~mm} \rrbracket$

$<5 \mathrm{~mm}^{\mathrm{k}}$

23

21

24

$5 \leq<15 \mathrm{~mm}^{\prime}$

25

26

22

$15 \leq<25 \mathrm{~mm}^{\mathrm{m}}$

2

3

4

The $P$ values among groups (keyhole approach group vs. Conventional craniotomy group (K/C); Keyhole approach group vs. Endovascular treatment group (K/E); Conventional craniotomy group vs.

Endovascular treatment group (C/E )) were: $a: P=0.51, P=0.48, P=0.17 ; b: P=0.68 ; P \llbracket 0.99 ; P=0.68 ; c$ :

$P=0.67, P=0.40, P=0.68$; d: $P=0.48, P=0.35, P=0.82$; $: P=0.73$, $P \otimes 0.99, P=0.73 ; f: P=0.69, P=0.84, P=0.84$; :

$P=0.60, P=0.81, P=0.44$; h: $P \bigotimes 0.99, P=0.63, P=0.63$; $: P=0.75, P \bigotimes 0.99, P=0.75 ; j: P=0.75, P \llbracket 0.99, P=0.75 ; k$ :

$P=0.69, P=0.84, P=0.55 ;$ I: $P=0.84, P=0.55, P=0.42 ; I: P=0.65, P=0.40, P=0.70$. 
No significant differences were found between the groups 
Table 2. Treatment Results of the Enrolled Patients

\section{Keyhole approach Conventional \\ Endovascular craniotomy group \\ group}

Total surgical time

$161.78 \pm 34.51$

$201.55 \pm 38.79$

$85.86 \pm 58.57$

(minutes) $^{\mathrm{a}}$

Cranial opening and closing time

$60.54 \pm 17.54$

$98.54 \pm 24.87$

None

(minutes) $^{\mathrm{b}}$

Time for aneurysm

$101.24 \pm 21.68$

$103.01 \pm 28.66$

$63.48 \pm 39.74$

treatment(minutes) ${ }^{c}$

Number of aneurysm ruptures

during surgery ${ }^{d}$

10

11

8

Length of postoperative

$11.42 \pm 6.64$

$18.03 \pm 7.14^{\mathrm{C}}$

$10.57 \pm 8.67$

hospitalization $^{\mathrm{e}}$

(days)

Hospitalization expenses

$(U S D)^{f}$

Glasgow score at discharge (cases)

5 points $^{9}$

40

38

40

4 points $^{h}$

4

3

3

3 points $^{\mathrm{i}}$

3

4

3

2 points ${ }^{j}$

1

2

1

1 point $^{k}$

2

3

3

Total cases

50

50

50

Complications

Cerebral vasospasm

1

2

2

Intracerebral infection

1

3

0

Hydrocephalus

2

3

4

Intracerebral hematomas

0

2

4

Epileptic seizures

0

4

0


The $P$ values among groups (keyhole approach group vs. Conventional craniotomy group(K/C); Keyhole approach group vs. Endovascular treatment group (K/E); Conventional craniotomy group vs.

Endovascular treatment group (C/E )) were: $a: P=0.002$, $P \llbracket 0.001, P \llbracket 0.001 ; b: P \llbracket 0.001 ; c: P=0.71, P \llbracket 0.001, F$ $\llbracket 0.001 ; d: P=0.81, P=0.60, P=0.44 ; e: P \llbracket 0.001, P=0.32, P \llbracket 0.001 ; f: P=0.03, P \llbracket 0.001, P \llbracket 0.001 ; g: P=0.009, P \rrbracket$ $0.99, P=0.35 ;$;: $P=0.63, P=0.08, P=0.63$; i: $P=0.70, P=0.70, P \llbracket 0.99 ; j: P=0.70, P \llbracket 0.99, P=0.70 ; k: P=0.56, P \rrbracket$ $0.99, P=0.56 ; \mathrm{l}: \mathrm{P}=0.65, \mathrm{P}=0.65, \mathrm{P} \otimes 0.99$.

\section{Figures}

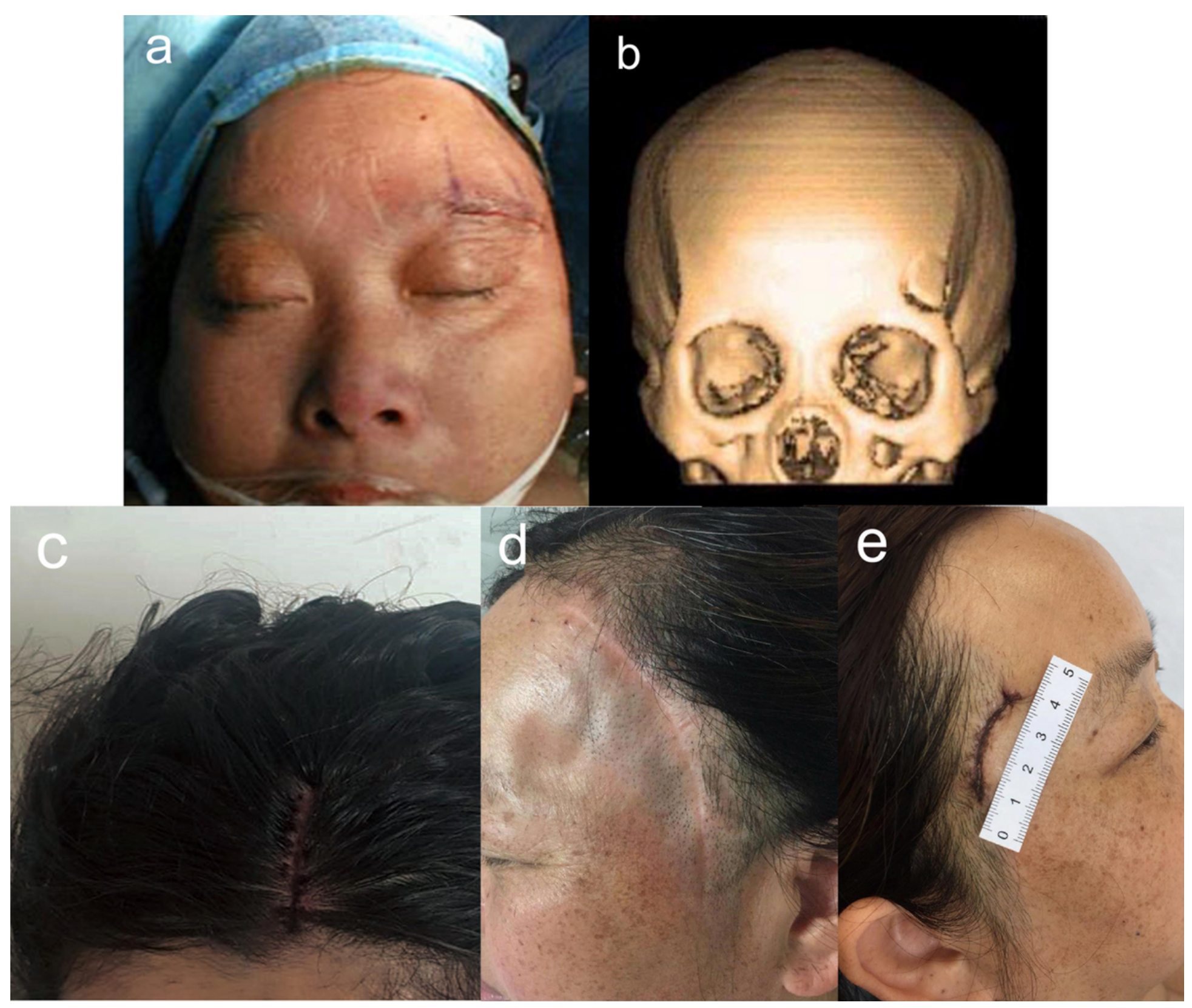

Figure 1 
The appearance of each surgical approach A. Supraorbital keyhole approach through a left eyebrow incision, B. CT reconstruction of the bone hole of supraorbital keyhole approach through a left eyebrow incision, C. Incision for trans-longitudinal fissure keyhole approach, D. Incision for conventional pterional approach, E. Incision for pterional keyhole approach. 